\title{
Chaos Induced by Snap-Back Repeller in a Two Species Competitive Model
}

\author{
Tahmineh Azizi, Bacim Alali \\ Department of Mathematics, Kansas State University, Manhattan, KS, USA \\ Email: tazizi@ksu.edu
}

How to cite this paper: Azizi, T. and Alali, B. (2020) Chaos Induced by Snap-Back Repeller in a Two Species Competitive Model. American Journal of Computational Mathematics, 10, 311-328.

https://doi.org/10.4236/ajcm.2020.102017

Received: June 1, 2020

Accepted: June 27, 2020

Published: June 30, 2020

Copyright $\odot 2020$ by author(s) and Scientific Research Publishing Inc. This work is licensed under the Creative Commons Attribution International License (CC BY 4.0).

http://creativecommons.org/licenses/by/4.0/

\begin{abstract}
In this paper, we investigate the complex dynamics of two-species Ricker-type discrete-time competitive model. We perform a local stability analysis for the fixed points and we will discuss about its persistence for boundary fixed points. This system inherits the dynamics of one-dimensional Ricker model such as cascade of period-doubling bifurcation, periodic windows and chaos. We explore the existence of chaos for the equilibrium points for a specific case of this system using Marotto theorem and proving the existence of snap-back repeller. We use several dynamical systems tools to demonstrate the qualitative behaviors of the system.
\end{abstract}

\section{Keywords}

Complex Dynamics, Snap-Back Repeller, Marotto Theorem, Persistence Theory, Bifurcation

\section{Introduction}

When we study the evolution of population dynamics, two major types of mathematical modelings can be used: the continuous-time dynamical systems and the discrete-time dynamical systems. For the purpose of modeling small size population and non-overlapping generations, the discrete time systems are the appropriate model [1]. There are so many studies that have been worked on discovering complex behaviors of discrete competitive model during the last decades [2] [3] [4] [5]. There are not many of these studies which are concerning about the existence of chaos in higher dimensional discrete dynamical systems. Chaos and chaos synchronizations have attracted many researchers for many years [6] [7]. In 1975, Li and York provided a simple criterion for chaos in one dimensional discrete dynamical systems, "period three implies chaos" [8]. This 
definition is the first description of chaos. Although, a precise definition of chaos was presented by their work, however, F.R. Marotto mentioned that the essential properties of chaos are the following: 1) there exist an infinite number of periodic solutions of various periods; 2) there exists an uncountably infinite set of points which exhibit random behavior; and 3) there is a highly sensitivity to initial conditions [9] [10] [11]. Marotto extended Li-York's chaos in one-dimension to multi-dimension through introducing the notion of snapback repeller by his famous theorem in 1978 a few years after Li and York definition for chaos. To explain more, we have mentioned the Marotto's definition for "Snap-back rappeler" and then his theorem [9]:

Definition 1.1 (Marotto-1978) Let $f$ be differentiable in $B_{r^{\prime}}(z)$. The point $z \in \mathbb{R}^{n}$ is an expanding fixed point of $f$ in $B_{r^{\prime}}(z)$, if $f(z)=z$ and all eigenvalues of $D f(x)$ exceed 1 in norm for all $x \in B_{r^{\prime}}(z)$.

Definition 1.2 (Marotto-1978) Assume that $z$ is an expanding fixed point of $f$ in $B_{r^{\prime}}(z)$ for some $r^{\prime}>0$. Then $z$ is said to be an snap-back repeller of $f$ if there exists a point $z_{0} \in B_{r^{\prime}}(z)$ with $z_{0} \neq z$ and $f^{M}\left(z_{0}\right)=z$ and $\left|D f^{M}\left(z_{0}\right)\right| \neq 0$ for some positive integer $M[9]$.

Figure 1 demonstrates the schematic diagram of snap-back repeller point.

Under the assumptions for definitions (1.1) and (1.2), the following theorem by Marotto holds.

Theorem 1.3 (Marotto-1978) If $f$ possesses a snap back repeller, then $f$ is chaotic in the following sense: There exist 1) a positive integer $N$, such that $f$ has a point of period $p$, for each integer $p \geq N, 2)$ a scrambled set of $f$, i.e., an uncountable set $S$ containing no periodic points of $f$, such that

a) $f(S) \subset S$,

b) $\limsup _{n \rightarrow \infty}\left\|f^{n}(x)-f^{n}(y)\right\|>0$, for all $x, y \in S$, with $x \neq y$,

c) $\limsup _{n \rightarrow \infty}\left\|f^{n}(x)-f^{n}(y)\right\|>0$, for all $x \in S$ and periodic point $y$ of $f$,

3) an uncountable subset $S_{0}$ of $S$, such that $\liminf _{n \rightarrow \infty}\left\|f^{n}(x)-f^{n}(y)\right\|=0$, for every $x, y \in S_{0} \quad$ [9].

However, there was a minor technical flaw in his work [11] [12] [13]. Although he wanted to apply his theorem to any repelling fixed point, some of the conditions that he considered in the proof of his theorem were associated with only expanding fixed points. He incorrectly mentioned that if the absolute value for all eigenvalues of $D f(z)$ is larger than 1 , then the fixed point $z$ is an expanding fixed point of $f$. As we know all expanding fixed points are repelling and its converse is not true. Therefore, Marotto definition for snap-back repeller and then his proof about existence of snap-back repeller implies chaos had a minor error. Chen et al., 1998; Lin et al., 2002; Li and Chen, 2003a; discussed about the flaws of Marotto's theorem and some of them provided several counterexamples to say that if all eigenvalues of the Jacobian $D f(z)$ at the fixed point $z$ are greater than one in norm, we cannot say always there exists some $s>1$ and $r^{\prime}>0$ such that for all $x, y \in B_{r^{\prime}}(z),\|f(x)-f(y)\|>s\|x-y\|$. Then they redefined the Marotto's Theorem as the following form [13]: 


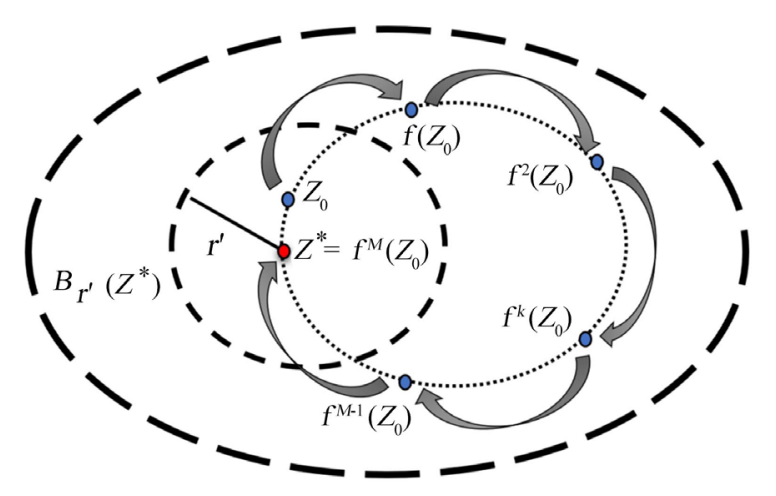

Figure 1. Snap-Back repeller schematic diagram.

Theorem 1.4 (Marotto-Li-Chen Theorem (2003)) Consider the following n-dimensional discrete dynamical system:

$$
x_{n+1}=f\left(x_{n}\right), x_{n} \in \mathbb{R}^{n}, n=0,1,2, \cdots
$$

where $f: \mathbb{R}^{n} \rightarrow \mathbb{R}^{n}$ and $z$ is a fixed point. Also assume that

1) $f(x)$ is continuously differentiable in $B_{r^{\prime}}(z)$ for some $r^{\prime}>0$,

2) All eigenvalues of $(D f(z))^{\mathrm{T}} D f(z)$ are greater than 1 ,

3) There exists a point $z_{0}=\left\{x \mid\|x-z\| \leq r^{\prime}\right.$ and all eigenvalues of $(D f(x))^{\mathrm{T}} D f(x)$ are larger than 1$\}$, with $z_{0} \neq z$, such that $f^{M}\left(z_{0}\right)=z$ where $f^{i}\left(z_{0}\right) \in B_{r^{\prime}}(z), i=0,1,2, \cdots, M$, and the determinant $\left|D f^{M}\left(z_{0}\right)\right| \neq 0$, for some positive integer $M$.

Then, the system is chaotic in the sense of Li-York [13].

Marotto refined his theorem in 2005 and he explained that a fixed point $z$ is called a repelling fixed point under differentiable function $f: \mathbb{R}^{n} \rightarrow \mathbb{R}^{n}$ if all eigenvalues of $D f(z)$ exceed 1 in magnitude, but $z$ is expanding only if

$$
\|f(x)-f(y)\|>s\|x-y\|
$$

where $s>1$, for all $x, y$ sufficiently close to $z$ with $x \neq y \quad\left(\right.$ for $\left.x, y \in B_{r^{\prime}}(z)\right)$. This implies that $f$ is a 1-1 function in $B_{r^{\prime}}(z)$ [11].

Definition 1.5 (Marotto-2005) Suppose $z$ is a fixed point of $f$ with all eigenvalues of $D f(z)$ exceeding 1 in magnitude and suppose that there exists a point $z_{0} \neq z$ in a repelling neighborhood of $z$ and an integer $M>1$, such that $x_{M}=z$ and $\operatorname{det}\left(D f\left(x_{k}\right)\right) \neq 0$ for $1 \leq k \leq M$ where $x_{k}=f^{k}\left(z_{0}\right)$. Then $z$ is called a snapback repeller of $f[11]$.

He claimed that since $\operatorname{det}\left(D f\left(x_{k}\right)\right) \neq 0$ for all $1 \leq k \leq M$, then the homoclinic orbit is transversal in the sense that $f$ for all $k \leq M$ is 1-1 map in a neighborhood of $x_{k}$.

As Marotto explained in 1978, the condition $\operatorname{det}\left(D f\left(x_{k}\right)\right) \neq 0$ guarantees the existence of the inverse of $f^{M}$ in $B_{r^{\prime}}(z)$. He mentioned that functions exhibit chaos and complex behavior when they possess snap-back repeller.

But what will happen that existence of a transverse homoclinic map convince us that we have chaos? As it is mentioned by many authors, a point which is in intersection of stable manifold and unstable manifold of a hyperbolic fixed point 
is called homoclinic point [10] [14] [15] [16] [17] [18]. If stable manifolds and unstable manifold of the hyperbolic fixed point, intersect transversally, then we have transverse homoclinic point in the intersection of both manifolds. In a neighborhood of a transverse homoclinic point, our map possesses an invariant cantor set on which it is topologically conjugate to a shift map. Shift map acting on the space of bi-infinite sequences of 0 's and 1's and it has the following properties:

A countable infinity of periodic orbits consists of orbits of all periods.

1) An uncountable infinity of non-periodic orbits.

2) A dense orbit.

Although, Wiggins in [16] mentioned that understanding the orbit structure of a map in that invariant Cantor set is impossible, he could show that the map in that invariant set behaves the same as shift map.

There are some researches which have more details about small neighborhood of a point on the homoclinic orbit [16] [19] [20] [21] [22]. The homoclinic orbits and homoclinic bifurcations which occur in continuous time dynamical systems has been studied widely by [23] [24] are using in discrete time systems by defining the Poincare map [15]. In 2011, L. Gardini et al. showed that critical homoclinic orbits lead to snap-back repellers and chaos too [15].

As Gardini et al. discussed, in non-invertible maps homoclinic orbits may be associate with expanding fixed points and or expanding cycles. Also, they mentioned that in the neighborhood of such homoclinic orbits, there exists an invariant set on which the map is chaotic. They even for the case that They proved that even if $\operatorname{det}\left(D f\left(x_{k}\right)\right)=0$, there are some situations in which the map is chaotic although Marotto theorem does not work. Laura et al., provide a definition for non-critical expanding fixed points and then they defined when a homoclinic orbit is critical. They used those definitions to prove a generalization of Marotto theorem in the case that we do not need the homoclinic orbit to be non-degenerate [15]:

Theorem 1.6 (L. Gardini. et al., (2011)) Let $f$ be a piecewise smooth non-invertible map, $f: X \rightarrow X, X \in \mathbb{R}^{n}$. Let $p$ be an expanding fixed point of $f$ and $O(p)$ a noncritical homoclinic orbit of $p$. Then in any neighborhood of $O(p)$, there exists an invariant cantor like set $\Lambda$ on which the fis chaotic [15].

In [14], Gardini studied the homoclinic bifurcations in n dimensional endomorphisms (maps with a nonunique inverse) which are associated to expanding periodic orbits. The study of chaos for these kinds of map in one dimension was studied by Mira in 1987 [25]. Since, this topic is out of the discussion for this paper, so we avoid going through that. In this paper, we study the local dynamics of a two-species Ricker competitive model with four biological parameters. We will conduct a local stability analysis to study the local dynamics of the steady states of the system. We will use the persistence theory to study the global dynamics of the system. To study the chaotic dynamics of the system, we focus on a specific case with only three biological parameters. We provide the condition 
under which Marotto theorem works for positive fixed points of this new system. Furthermore, this model does not have a Neimark-Sacker bifurcation and inherits the same dynamics as one dimensional Ricker model. We will numerically demonstrate the local and qualitative dynamics of the system using several dynamical system tools.

\section{The Two-Species Ricker Competitive Model and Its Local Dynamics}

The Ricker model is a well known population model which demonstrates stable, periodic and non-periodic and complex nonlinear dynamics [26] [27]. Here, we consider a two-species Ricker model which is a special case of model (2) in [3] and has the following form:

$$
\begin{gathered}
f_{1}=X_{1}(n+1)=X_{1}(n) \mathrm{e}^{n\left(1-\frac{X_{1}(n)}{k}-X_{2}(n)\right)} \\
f_{2}=X_{2}(n+1)=X_{2}(n) \mathrm{e}^{r_{2}\left(1-\frac{X_{2}(n)}{l}-X_{1}(n)\right)}
\end{gathered}
$$

here, $X_{1}$ demonstrates the population size of the first species, $X_{2}$ represents the population size of the second species, $r_{1}$ and $r_{2}$ are the intrinsic growth rate, $k$ and $l$ the carrying capacity of the environment.

The Jacobian matrix for (1)-(2) has the form

$$
J:=\left[\begin{array}{ll}
\frac{\partial f_{1}}{\partial X_{1}} & \frac{\partial f_{1}}{\partial X_{2}} \\
\frac{\partial f_{2}}{\partial X_{1}} & \frac{\partial f_{2}}{\partial X_{2}}
\end{array}\right]
$$

where

$$
\begin{gathered}
\frac{\partial f_{1}}{\partial X_{1}}=\left(1-\frac{r_{1} X_{1}}{k}\right) \exp \left(r_{1}\left(1-\frac{X_{1}}{k}-X_{2}\right)\right) \\
\frac{\partial f_{1}}{\partial X_{2}}=-r_{1} X_{1} \exp \left(r_{1}\left(1-\frac{X_{1}}{k}-X_{2}\right)\right) \\
\frac{\partial f_{2}}{\partial X_{1}}=-r_{2} X_{2} \exp \left(r_{2}\left(1-\frac{X_{2}}{l}-X_{1}\right)\right) \\
\frac{\partial f_{2}}{\partial X_{2}}=\left(1-\frac{r_{2} X_{2}}{l}\right) \exp \left(r_{2}\left(1-\frac{X_{2}}{l}-X_{1}\right)\right)
\end{gathered}
$$

Then, at the origin we have

$$
\left.J\right|_{(0,0)}=\left(\begin{array}{cc}
\mathrm{e}^{r_{1}} & 0 \\
0 & \mathrm{e}^{r_{2}}
\end{array}\right)
$$

and for the fixed point $(k, 0)$ we have

$$
\left.J\right|_{(k, 0)}=\left(\begin{array}{cc}
1-r_{1} & -k r_{1} \\
0 & \mathrm{e}^{r_{2}(1-l)}
\end{array}\right)
$$

and for the fixed point $(0, l)$ we have 


$$
\left.J\right|_{(0, l)}=\left(\begin{array}{cc}
\mathrm{e}^{r_{1}(1-k)} & 0 \\
-l r_{2} & 1-r_{2}
\end{array}\right)
$$

and for the positive fixed point $\left(X_{1}^{*}, X_{2}^{*}\right)=\left(\frac{k(1-l)}{1-k l}, \frac{l(1-k)}{1-k l}\right)$, we have

$$
\left.J\right|_{\left(X_{1}^{*}, X_{2}^{*}\right)}=\left(\begin{array}{cc}
\frac{-1+k l+r_{1}-r_{1} l}{-1+k l} & \frac{-k(-1+l) r_{1}}{-1+k l} \\
\frac{-k(-1+k) r_{2}}{-1+k l} & \frac{-1+k l+r_{2}-r_{2} k}{-1+k l}
\end{array}\right)
$$

Proposition 2.1 The local stability analysis results for the fixed points $(0,0)$, $(k, 0),(0, l)$ of $(1)-(2)$ are summarized as below.

1) The equilibrium point $(0,0)$ is always an unstable fixed point.

2) The equilibrium point $(k, 0)$ for $l<1$ and $0<r_{1}<2$, has a stable manifold in $X_{1}$ direction and an unstable manifold in $X_{2}$ direction and is a saddle point. Also, $(k, 0)$ for $l>1$ and $0<r_{1}<2$, has a stable manifold in $X_{1}$ direction and a stable manifold in $X_{2}$ direction and is a stable node. Moreover, $(k, 0)$ for $l<1$ and $r_{1}>2$, has an unstable manifold in $X_{1}$ direction and an unstable manifold in $X_{2}$ direction and is an unstable node. Finally, $(k, 0)$ for $l>1$ and $r_{1}>2$, has an unstable manifold in $X_{1}$ direction and a stable manifold in $X_{2}$ direction and is a saddle point.

3) The equilibrium point $(0, l)$ for $k<1$ and $0<r_{1}<2$, has a stable manifold in $X_{2}$ direction and an unstable manifold in $X_{1}$ direction and is a saddle point. Also, $(0, l)$ for $k>1$ and $0<r_{2}<2$, has a stable manifold in $X_{1}$ direction and a stable manifold in $X_{2}$ direction and is a stable node. Moreover, $(0, l)$ for $k<1$ and $r_{2}>2$, has an unstable manifold in $X_{1}$ direction and an unstable manifold in $X_{2}$ direction and is an unstable node. Finally, $(0, l)$ for $k>1$ and $r_{1}>2$, has an unstable manifold in $X_{2}$ direction and a stable manifold in $X_{1}$ direction and is a saddle point.

\section{Global Stability Analysis Using Persistence Theory}

\subsection{Boundedness of the System Solutions}

To study the global stability of the equilibrium points of system, at first we prove that all solutions in the first quadrant $\mathbb{R}_{+}^{2}$ are eventually bounded.

Theorem 3.1 For $r_{1}, r_{2}>0, k, l>0$ and initial conditions in the first quadrant $\mathbb{R}_{+}^{2}$, i.e. $X_{1}(0)>0$ and $X_{2}(0)>0$, for the system of (1)-(2) we have: $X_{1}>0$ and $X_{2}>0$ for all $n \in \mathbb{Z}^{+}$. In addition, we can find some positive number $M$, such that $\max _{n \in \mathbb{Z}^{+}}\left\{X_{1}(n), X_{2}(n)\right\} \leq M$.

Proof. By induction.

Since $X_{1}(0)>0$ we have $\exp \left(r_{1}\left(1-\frac{X_{1}(0)}{k}\right)\right)>0$, hence

$$
X_{1}(1)=X_{1}(0) \mathrm{e}^{r_{1}\left(1-\frac{X_{1}(0)}{k}-X_{2}(0)\right)}<X_{1}(0) \mathrm{e}^{r_{1}\left(1-\frac{X_{1}(0)}{k}\right)}>0
$$


Assume that for $n \leq j$, we have $X_{1}(j)>0$. Then for $n=j+1$ we have

$$
X_{1}(j+1)=X_{1}(j) \mathrm{e}^{\eta\left(1-\frac{X_{1}(j)}{k}-X_{2}(j)\right)}>0
$$

Therefore $X_{1}(n)>0$ for any $n \in \mathbb{Z}^{+}$. Similarly, since $X_{1}(0)>0$ and $X_{2}(0)>0$, we automatically have $\exp \left(r_{2}\left(1-\frac{X_{2}(0)}{j}\right)\right)>0$ is positive. Hence,

$$
X_{2}(1)=X_{2}(0) \mathrm{e}^{r_{2}\left(1-\frac{X_{2}(0)}{j}-X_{1}(0)\right)}<X_{2}(0) \mathrm{e}^{r_{2}\left(1-\frac{X_{2}(0)}{j}\right)}>0
$$

Assume that for $n \leq j$, we have $X_{2}(j)>0$. Then for $n=j+1$ we have

$$
X_{2}(j+1)=X_{2}(j) \mathrm{e}^{r_{2}\left(1-\frac{X_{2}(l)}{j}-X_{1}(j)\right)}>0
$$

Therefore $X_{2}(n)>0$ for any $n \in \mathbb{Z}^{+}$.

To find an upper bound, we know,

$$
X_{1}(n+1)=X_{1}(n) \mathrm{e}^{n\left(1-\frac{X_{1}(n)}{k}\right)} \leq \max _{x \in \mathbb{R}^{+}}\{f(x)\}
$$

If we define $f_{1}(x)=x \mathrm{e}^{\eta\left(1-\frac{x}{k}\right)}$, then $f_{1}^{\prime}(x)=\left(1-\frac{r_{1} x}{k}\right) \mathrm{e}^{\eta\left(1-\frac{x}{k}\right)}$ and $f_{1}(x)$ has critical points at $x=\frac{k}{r_{1}}$. Since $f_{1}^{\prime}(x)>0$ if $x<\frac{k}{r_{1}}$ and $f_{1}^{\prime}(x)<0$ if $x>\frac{k}{r_{1}}$, then $x=\frac{k}{r_{1}}$ is the maximal point of $f_{1}(x)$, i.e. $\max _{x \in \mathbb{R}^{+}}\left\{f_{1}(x)\right\}=f_{1}\left(\frac{k}{r_{1}}\right)$. Hence,

$$
x_{1}(n+1)=X_{1}(n) \mathrm{e}^{\eta\left(1-\frac{X_{1}(n)}{k}-X_{2}(n)\right)} \leq f_{1}\left(\frac{k}{r_{1}}\right)=\frac{k \mathrm{e}^{r_{1}-1}}{r_{1}}=M_{1}
$$

Similarly, we define $f_{2}(x)=x \mathrm{e}^{r_{2}\left(1-\frac{x}{l}\right)}$, then $f_{2}^{\prime}(x)=\left(1-\frac{r_{2} x}{l}\right) \mathrm{e}^{r_{2}\left(1-\frac{x}{l}\right)}$ and $f_{2}(x)$ has critical points at $x=\frac{l}{r_{2}}$. Since $f_{2}^{\prime}(x)>0$ if $x<\frac{l}{r_{2}}$ and $f_{2}^{\prime}(x)<0$ if $x>\frac{l}{r_{2}}$, then $x=\frac{l}{r_{2}}$ is the maximal point of $f_{2}(x)$, i.e. $\max _{x \in \mathbb{R}^{+}}\left\{f_{2}(x)\right\}=f_{2}\left(\frac{l}{r_{2}}\right)$.

$$
X_{2}(n+1)=X_{2}(n) \mathrm{e}^{r_{2}\left(1-\frac{X_{2}(n)}{l}-X_{1}(n)\right)} \leq f_{2}\left(\frac{l}{r_{2}}\right)=\frac{l \mathrm{e}^{r_{2}-1}}{r_{2}}=M_{2}
$$

Therefore, we can find some positive number $M=\max \left\{M_{1}, M_{2}\right\}$, such that $\max _{n \in \mathbb{Z}^{+}}\left\{X_{1}(n), X_{2}(n)\right\} \leq M$.

\subsection{Persistence of the Species}

To work on global stability, we need to study the persistence theory [28] [29]. 
Here, we consider two cases:

1) Persistence of system corresponding to $(k, 0)$.

2) Persistence of system corresponding to $(0, l)$.

\subsubsection{Case 1: Persistence of System Corresponding to $(k, 0)$}

For the first case, we have:

$$
\begin{gathered}
P=\left\{\left(X_{1}, X_{2}\right): X_{1} \geq 0, X_{2} \geq 0\right\} \\
P_{k, 0}=\left\{\left(X_{1}, X_{2}\right) \in P: X_{1}>0\right\} \\
\partial P_{k, 0}=P \backslash P_{k, 0}
\end{gathered}
$$

Proposition 3.2 The system is uniformly persistent with respect to $\left(P_{k, 0}, \partial P_{k, 0}\right)$.

Proof. Here, $\partial P_{k, 0}$ is closed in $P$. For any positive solution of $\left(X_{1}(n), X_{2}(n)\right)$ of the system, as we proved in theorem (3.1), we have

$$
X_{1}(n+1) \leq X_{1}(n) \mathrm{e}^{n\left(1-\frac{X_{1}(n)}{k}\right)} \leq \max _{X_{1} \in \mathbb{R}^{+}}\left\{f_{1}\left(X_{1}\right)\right\}=\frac{k \mathrm{e}^{r_{1}-1}}{r_{1}}=M_{1}
$$

And for large enough $n$

$$
X_{2}(n+1) \leq X_{2}(n) \mathrm{e}^{r_{2}\left(1-\frac{X_{2}(n)}{l}\right)} \leq \max _{X_{2} \in \mathbb{R}^{+}}\left\{f_{2}\left(X_{2}\right)\right\}=\frac{l \mathrm{e}^{r_{2}-1}}{r_{2}}=M_{2}
$$

Therefore, system (1)-(2) is point dissipative. Assume for all $n \geq 0$

$$
\begin{aligned}
Y_{\partial}=\{( & \left.X_{1}(0), X_{2}(0)\right):\left(X_{1}(n), X_{2}(n)\right) \text { satisfies the system equations } \\
& \text { and } \left.\left(X_{1}(n), X_{2}(n)\right) \in \partial P_{k, 0}\right\}
\end{aligned}
$$

We see that

$$
Y_{\partial}=\left\{\left(0, X_{2}\right): X_{2} \geq 0\right\}=\partial P_{k, 0}
$$

Moreover, $(0,0)$ is the unique equilibrium in $Y_{\partial}$. Define $W^{s}(0,0)$ to be the stable manifold for $(0,0)$. We show that

$$
W^{s}(0,0) \cap P_{k, 0}=\varnothing
$$

Assume that in contradiction, there exist a solution $\left(X_{1}(n), X_{2}(n)\right)$ of system with $X_{1}(n)>0$ such that

$$
\left(X_{1}(n), X_{2}(n)\right) \rightarrow(0,0) \text { as } n \rightarrow \infty
$$

Then, for large $n$ we have

$$
X_{1}(n+1)>X_{1}(n) \mathrm{e}^{r_{1} / 2}
$$

Since $r_{1}>0$, it follows that $X_{1}(n) \rightarrow \infty$ as $n \in \infty$ and contradiction. Also, every orbit in $Y_{\partial}$ tends to $(0,0)$ as $n \rightarrow \infty$. It means that $(0,0)$ is an isolated invariant set in $P$ and acyclic in $Y_{\partial}$. Note that $Y_{\partial}$ repels uniformly the solution of systems with positive $X_{1}(n)$ [30] [31]. It follows that there is $s_{1}>0$ such that $X_{1}(n)>s_{1}$ for large enough $n$. 
Theorem 3.3. There exists $s_{1}>0$ such that for any $X_{1}(0)>0$ we have

$$
s_{1}<X_{1}(n)<\frac{k \mathrm{e}^{\eta_{1}-1}}{r_{1}}
$$

Proof. By proposition (3.2).

Theorem 3.4 All solutions $\left\{\left(X_{1}(n), X_{2}(n)\right)\right\}$ of system with $X_{1}(0)>0$ and $X_{2}(0) \geq 0$, for $l>1$ and $0<r_{1}<2$, are decreasing to the fixed point $(k, 0)$, i.e.

$$
\lim _{n \rightarrow+\infty} X_{1}(n)=k, \quad \lim _{n \rightarrow+\infty} X_{2}(n)=0
$$

Proof. By proposition (3.2) and theorem (3.3).

\subsubsection{Case 2: Persistence of System Corresponding to $(0, I)$}

For this case, we have:

$$
\begin{gathered}
Q=\left\{\left(X_{1}, X_{2}\right): X_{1} \geq 0, X_{2} \geq 0\right\} \\
Q_{0, l}=\left\{\left(X_{1}, X_{2}\right) \in Q: X_{2}>0\right\} \\
\partial Q_{0, l}=Q \backslash Q_{0, l}
\end{gathered}
$$

Proposition 3.5 The system is uniformly persistent with respect to $\left(Q_{0, l}, \partial Q_{0, l}\right)$.

Proof. Here, $\partial Q_{0, l}$ is closed in $Q$. Similarly, for any positive solution of $\left(X_{1}(n), X_{2}(n)\right)$ of the system (1)-(2), similar to theorem (3.1), we can write

$$
X_{1}(n+1) \leq X_{1}(n) \mathrm{e}^{n\left(1-\frac{X_{1}(n)}{k}\right)} \leq \max _{X_{1} \in \mathbb{R}^{+}}\left\{f\left(X_{1}\right)\right\}=\frac{k \mathrm{e}^{r_{1}-1}}{r_{1}}=M_{1}
$$

For large enough $n$

$$
X_{2}(n+1) \leq X_{2}(n) \mathrm{e}^{r_{2}\left(1-\frac{X_{2}(n)}{l}\right)} \leq \max _{X_{2} \in \mathbb{R}^{+}}\left\{f\left(X_{2}\right)\right\}=\frac{l \mathrm{e}^{r_{2}-1}}{r_{2}}=M_{2}
$$

Thus, system (1)-(2) is point dissipative. Now, for all $n \geq 0$, we set

$$
\begin{aligned}
L_{\partial}= & \left\{\left(X_{1}(0), X_{2}(0)\right):\left(X_{1}(n), X_{2}(n)\right)\right. \text { satisfies the systeme quations } \\
& \text { and } \left.\left(X_{1}(n), X_{2}(n)\right) \in \partial Q_{0, l}\right\}
\end{aligned}
$$

for which

$$
L_{\partial}=\left\{\left(X_{1}, 0\right): X_{2} \geq 0\right\}=\partial Q_{0, l}
$$

Moreover, $(0,0)$ is the unique equilibrium in $L_{\partial}$. Set $W^{s}(0,0)$ to be the stable manifold for $(0,0)$. We prove that

$$
W^{s}(0,0) \cap Q_{0, l}=\varnothing
$$

By contradiction, there exist a solution $\left(X_{1}(n), X_{2}(n)\right)$ of system with $X_{2}(n)>0$ such that

$$
\left(X_{1}(n), X_{2}(n)\right) \rightarrow(0,0) \text { as } n \rightarrow \infty
$$

For large $n$ we have 


$$
X_{2}(n+1)>X_{2}(n) \mathrm{e}^{r_{2} / 2}
$$

Since $r_{2}>0$, it leads to $X_{2}(n) \rightarrow \infty$ as $n \in \infty$ which is a contradiction. Also, every orbit in $L_{\partial}$ tends to $(0,0)$ as $n \rightarrow \infty$. It implies that $(0,0)$ is an isolated invariant set in $Q$ and acyclic in $L_{\partial}$. Here, $l_{\partial}$ repels uniformly the solutions of system with positive $X_{2}(n)$ [30] [31]. It follows that there is $s_{2}>0$ such that $X_{2}(n)>s_{2}$ for large enough $n$.

Theorem 3.6 There exists $s_{2}>0$ such that for any $X_{2}(0)>0$ we have

$$
s_{2}<X_{2}(n)<\frac{l \mathrm{e}^{r_{2}-1}}{r_{2}}
$$

Proof. By proposition (3.5).

Theorem 3.7 All solutions $\left\{\left(X_{1}(n), X_{2}(n)\right)\right\}$ of system with $X_{1}(0) \geq 0$ and $X_{2}(0)>0$, for $k>1$ and $0<r_{2}<2$, are decreasing to the fixed point $(0, l)$, i.e.

$$
\lim _{n \rightarrow+\infty} X_{1}(n)=0, \quad \lim _{n \rightarrow+\infty} X_{2}(n)=l
$$

Proof. By proposition (3.5) and theorem (3.6).

Finally, we have the following result

Theorem 3.8 If there are positive constants $s_{1}, s_{2}>0$ and $M_{1}, M_{2}>0$ such that the solution $\left(X_{1}(n), X_{2}(n)\right)$ of system satisfies

$$
\begin{aligned}
& 0<s_{1} \leq \lim _{n \rightarrow+\infty} \inf X_{1}(n) \leq \lim _{n \rightarrow+\infty} \sup X_{1}(n) \leq M_{1}=\frac{k \mathrm{e}^{n_{1}-1}}{r_{1}} \\
& 0<s_{2} \leq \lim _{n \rightarrow+\infty} \inf X_{2}(n) \leq \lim _{n \rightarrow+\infty} \sup X_{2}(n) \leq M_{2}=\frac{l \mathrm{e}^{r_{2}-1}}{r_{2}}
\end{aligned}
$$

Then, system (1)-(2) is persistent. If system is not persistent, it is called non-persistent smith2011dynamical.

\section{Application of Snap-Back Repeller and Marroto Chaos in Study of Chaotic Dynamics of System}

In this section, we explore analytically chaos in the sense of Marotto for a specific case of model (1)-(2). Without loss of generality, we consider $k=l$, then we have

$$
F:=\left\{\begin{array}{l}
g_{1}\left(X_{1}(n), X_{2}(n)\right)=X_{1}(n) \exp \left(r_{1}\left(1-\frac{X_{1}(n)}{k}-X_{2}(n)\right)\right) \\
g_{2}\left(X_{1}(n), X_{2}(n)\right)=X_{2}(n) \exp \left(r_{2}\left(1-\frac{X_{2}(n)}{k}-X_{1}(n)\right)\right)
\end{array}\right.
$$

The Jacobian matrix for (5) has the form

$$
J:=\left[\begin{array}{ll}
\frac{\partial g_{1}}{\partial X_{1}} & \frac{\partial g_{1}}{\partial X_{2}} \\
\frac{\partial g_{2}}{\partial X_{1}} & \frac{\partial g_{2}}{\partial X_{2}}
\end{array}\right]
$$


where

$$
\begin{gathered}
\frac{\partial g_{1}}{\partial X_{1}}=\left(1-\frac{r_{1} X_{1}}{k}\right) \exp \left(r_{1}\left(1-\frac{X_{1}}{k}-X_{2}\right)\right) \\
\frac{\partial g_{1}}{\partial X_{2}}=-r_{1} X_{1} \exp \left(r_{1}\left(1-\frac{X_{1}}{k}-X_{2}\right)\right) \\
\frac{\partial g_{2}}{\partial X_{1}}=-r_{2} X_{2} \exp \left(r_{2}\left(1-\frac{X_{2}}{k}-X_{1}\right)\right) \\
\frac{\partial g_{2}}{\partial X_{2}}=\left(1-\frac{r_{2} X_{2}}{k}\right) \exp \left(r_{2}\left(1-\frac{X_{2}}{k}-X_{1}\right)\right)
\end{gathered}
$$

For this specific case, we have four fixed points $(0,0),(k, 0),(0, k)$ and $\left(X_{1}^{*}, X_{2}^{*}\right)=\left(\frac{k}{k+1}, \frac{k}{k+1}\right)$. At $(0,0)$ we have

$$
\left.J\right|_{(0,0)}=\left(\begin{array}{cc}
\mathrm{e}^{r_{1}} & 0 \\
0 & \mathrm{e}^{r_{2}}
\end{array}\right)
$$

and at $(k, 0)$ we have

$$
\left.J\right|_{(k, 0)}=\left(\begin{array}{cc}
1-r_{1} & -k r_{1} \\
0 & \mathrm{e}^{r_{2}(1-k)}
\end{array}\right)
$$

and also for the fixed point $(0, k)$ we have

$$
\left.J\right|_{(0, k)}=\left(\begin{array}{cc}
\mathrm{e}^{r_{1}(1-k)} & 0 \\
-k r_{2} & 1-r_{2}
\end{array}\right)
$$

and finally for the positive fixed point $\left(X_{1}^{*}, X_{2}^{*}\right)=\left(\frac{k}{k+1}, \frac{k}{k+1}\right)$, we have

$$
\left.J\right|_{\left(X_{1}^{*}, X_{2}^{*}\right)}=\left(\begin{array}{cc}
\frac{k+1-r_{1}}{k+1} & \frac{-k r_{1}}{k+1} \\
\frac{-k r_{2}}{k+1} & \frac{k+1-r_{2}}{k+1}
\end{array}\right)
$$

where

$$
\begin{gathered}
\operatorname{det}\left(\left.J\right|_{\left(X_{1}^{*}, X_{2}^{*}\right)}\right)=-\frac{k r_{1} r_{2}-r_{1} r_{2}-k+r_{1}+r_{2}-1}{k+1} \\
\operatorname{tr}\left(\left.J\right|_{\left(X_{1}^{*}, X_{2}^{*}\right)}\right)=\frac{2 k+2-r_{2}-r_{1}}{k+1}
\end{gathered}
$$

and also, characteristic polynomial has the form

$$
P(X):=X^{2}-\frac{2 k+2-r_{2}-r_{1}}{k+1} X-\frac{k r_{1} r_{2}-r_{1} r_{2}-k+r_{1}+r_{2}-1}{k+1}
$$

Proposition 4.1 The local stability analysis results for the fixed points $(0,0)$, $(k, 0),(0, k)$ of $(5)$ are summarized as below.

1) The equilibrium point $(0,0)$ is always an unstable fixed point.

2) The equilibrium point $(k, 0)$ for $k<1$ and $0<r<2$, has a stable ma- 
nifold in $X_{1}$ direction and an unstable manifold in $X_{2}$ direction and is a saddle point. Also, $(k, 0)$ for $k>1$ and $0<r<2$, has a stable manifold in $X_{1}$ direction and a stable manifold in $X_{2}$ direction and is a stable node. Moreover, $(k, 0)$ for $k<1$ and $r>2$, has an unstable manifold in $X_{1}$ direction and an unstable manifold in $X_{2}$ direction and is an unstable node. Finally, $(k, 0)$ for $k>1$ and $r>2$, has an unstable manifold in $X_{1}$ direction and a stable manifold in $X_{2}$ direction and is a saddle point.

3) The equilibrium point $(0, k)$ for $k<1$ and $0<r<2$, has a stable manifold in $X_{2}$ direction and an unstable manifold in $X_{1}$ direction and is a saddle point. Also, $(0, k)$ for $k>1$ and $0<r<2$, has a stable manifold in $X_{1}$ direction and a stable manifold in $X_{2}$ direction and is a stable node. Moreover, $(0, k)$ for $k<1$ and $r>2$, has an unstable manifold in $X_{1}$ direction and an unstable manifold in $X_{2}$ direction and is an unstable node. Finally, $(0, k)$ for $k>1$ and $r>2$, has an unstable manifold in $X_{2}$ direction and a stable manifold in $X_{1}$ direction and is a saddle point.

Proposition 4.2 The local stability analysis results for the fixed points $\left(X_{1}^{*}, X_{2}^{*}\right)=\left(\frac{k}{k+1}, \frac{k}{k+1}\right)$ of $(5)$ are summarized as below.

1) The equilibrium point $\left(X_{1}^{*}, X_{2}^{*}\right)$ is an unstable fixed point if and only if $r_{1} r_{2}(1-k)+2(k+1)<\left(r_{1}+r_{2}\right), 4(k+1)-2\left(r_{1}+r_{2}\right)+r_{1} r_{2}(1-k)>0, k<1$

or

$$
k<\frac{r_{1} r_{2}-r_{1}-r_{2}}{r_{1} r_{2}}, 4(k+1)-2\left(r_{1}+r_{2}\right)+r_{1} r_{2}(1-k)>0, k<1
$$

2) The equilibrium point $\left(X_{1}^{*}, X_{2}^{*}\right)$ is a stable fixed point if and only if

$$
k>\frac{r_{1} r_{2}-r_{1}-r_{2}}{r_{1} r_{2}}, 4(k+1)-2\left(r_{1}+r_{2}\right)+r_{1} r_{2}(1-k)>0, k<1
$$

3) The equilibrium point $\left(X_{1}^{*}, X_{2}^{*}\right)$ is a saddle point if and only if

$$
4(k+1)-2\left(r_{1}+r_{2}\right)+r_{1} r_{2}(1-k)<0, k>1
$$

Proof. Using Theorem 1.1.1 (Linearized Stability) in [32].

The equilibrium point $\left(X_{1}^{*}, X_{2}^{*}\right)$ is an unstable fixed point if and only if $|\operatorname{det}(J)|>1 \quad$ and $\quad|\operatorname{tr}(J)|<|1+\operatorname{det}(J)| \quad \cdot \operatorname{tr}\left(\left.J\right|_{\left(X_{1}^{*}, X_{2}^{*}\right)}\right)-\operatorname{det}\left(\left.J\right|_{\left(X_{1}^{*}, X_{2}^{*}\right)}\right)-1<0$ gives us:

$$
\frac{r_{1} r_{2}(k-1)}{k+1}<0 \rightarrow k<1
$$

Also, $\operatorname{tr}\left(\left.J\right|_{\left(X_{1}^{*}, X_{2}^{*}\right)}\right)+\operatorname{det}\left(\left.J\right|_{\left(X_{1}^{*}, X_{2}^{*}\right)}\right)+1<0$ gives us:

$$
\frac{4(k+1)-2\left(r_{1}+r_{2}\right)+r_{1} r_{2}(1-k)}{k+1}>0
$$

and $\operatorname{det}\left(\left.J\right|_{\left(X_{1}^{*}, X_{2}^{*}\right)}\right)>1$ gives us 


$$
\frac{r_{1} r_{2}(1-k)-\left(r_{1}+r_{2}\right)}{k+1}<0
$$

that is to say

$$
k>\frac{r_{1} r_{2}-r_{1}-r_{2}}{r_{1} r_{2}}
$$

Moreover, $\operatorname{det}\left(\left.J\right|_{\left(X_{1}^{*}, X_{2}^{*}\right)}\right)<-1$ gives us

$$
\frac{r_{1} r_{2}(1-k)+2(k+1)-\left(r_{1}+r_{2}\right)}{k+1}<0
$$

The positive fixed point of system (5) is asymptotically stable if and only if

$$
|\operatorname{tr}(J)|<1+\operatorname{det}(J)<2
$$

We check (17) using (12) and (13). $\operatorname{tr}\left(\left.J\right|_{\left(X_{1}^{*}, X_{2}^{*}\right)}\right)-\operatorname{det}\left(\left.J\right|_{\left(X_{1}^{*}, X_{2}^{*}\right)}\right)-1<0$ and $\operatorname{tr}\left(\left.J\right|_{\left(X_{1}^{*}, X_{2}^{*}\right)}\right)+\operatorname{det}\left(\left.J\right|_{\left(X_{1}^{*}, X_{2}^{*}\right)}\right)+1<0 \quad$ give us $\quad$ (15) and (16) and $\operatorname{det}\left(\left.J\right|_{\left(X_{1}^{*}, X_{2}^{*}\right)}\right)<1$ gives us

$$
\frac{r_{1} r_{2}(1-k)-\left(r_{1}+r_{2}\right)}{k+1}<0
$$

that is to say

$$
k>\frac{r_{1} r_{2}-r_{1}-r_{2}}{r_{1} r_{2}}
$$

Finally, The equilibrium point $\left(X_{1}^{*}, X_{2}^{*}\right)$ is a saddle point if and only if $\operatorname{tr}^{2}(J)-4 \operatorname{det}(J)>0$ and $|\operatorname{tr}(J)|>|1+\operatorname{det}(J)|$. The first condition gives us

$$
\frac{\left(r_{1}-r_{2}\right)^{2}+4 k^{2}}{(k+1)^{2}}>0
$$

which is always true. Another conditions to check are: $\operatorname{tr}\left(\left.J\right|_{\left(X_{1}^{*}, X_{2}^{*}\right)}\right)-\operatorname{det}\left(\left.J\right|_{\left(X_{1}^{*}, X_{2}^{*}\right)}\right)-1>0$ gives us:

$$
\frac{r_{1} r_{2}(k-1)}{k+1}>0
$$

and, $\operatorname{tr}\left(\left.J\right|_{\left(X_{1}^{*}, X_{2}^{*}\right)}\right)+\operatorname{det}\left(\left.J\right|_{\left(X_{1}^{*}, X_{2}^{*}\right)}\right)+1<0$ which gives us:

$$
\frac{4(k+1)-2\left(r_{1}+r_{2}\right)+r_{1} r_{2}(1-k)}{k+1}<0
$$

Numerical simulations, including bifurcation diagrams and time series display that this model demonstrates chaotic oscillations after a cascade of period-doubling bifurcations. As we can see in Figure 2, there are chaotic regions which are embedded in periodic windows regions. The periodic behaviors which appear alternately in the chaotic area, contain a copy of bifurcation diagram and 
it is repeating when we are changing the bifurcation parameter $r$. The bifurcation diagram for system (5) with respect to $r$ displays the same qualitative dynamics for different values of $k$. Moreover, we have run bifurcation analysis with respect to $k$ with different $r$ values in Figure 3.

Also, if we look at Figure 4, at first, the equilibrium point is stable, when we increase $r$, it loses stability, from one cycle to two cycles, and produces a flip bifurcation. As $r$ continues to increase, periodic oscillations are observed with periods $4, \ldots$, which eventually leads to chaos.

To prove the existence of chaos for the map (5) in the sense of Marotto, we need to find the conditions under which the fixed point $Z^{*}=\left(X_{1}^{*}, X_{2}^{*}\right)$ of the system is a snap-back repeller. According to definition (1.5) and Figure 1, we need to find a neighborhood $B_{r^{\prime}}\left(Z^{*}\right)$ of $Z^{*}$ in which all eigenvalues have absolute value more than one. Now, we give the following lemma which we need that to prove chaos in the sense of Marotto for positive fixed point $Z^{*}=\left(X_{1}^{*}, X_{2}^{*}\right)$ of map (5).
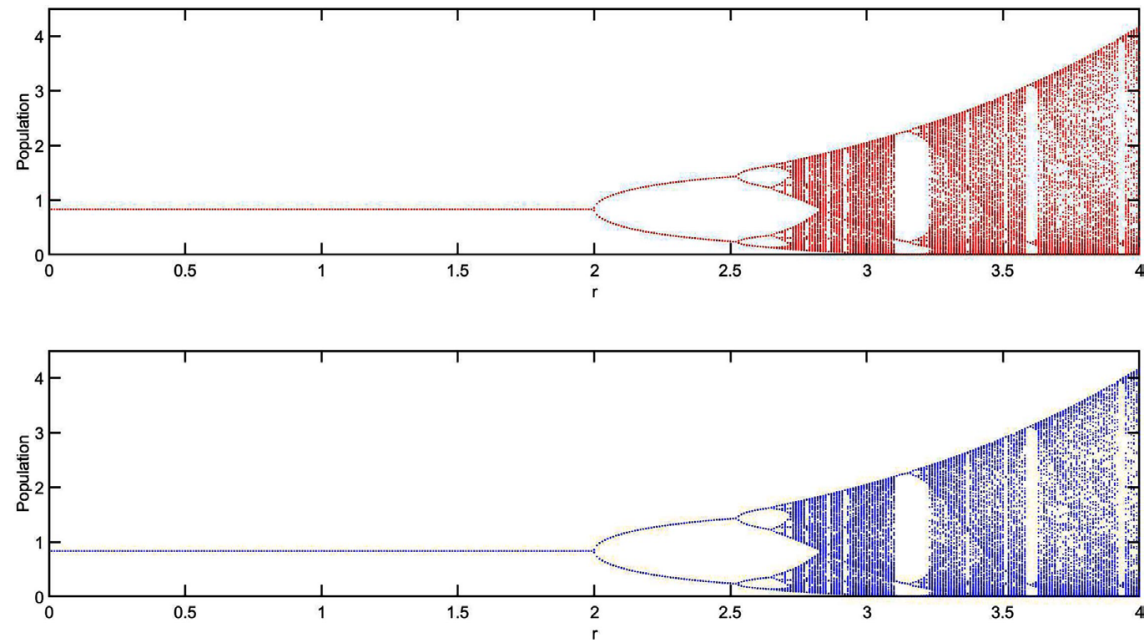

Figure 2. Bifurcation diagram of system (4.1) when $k=10$ and $r_{1}=r_{2}=r$.
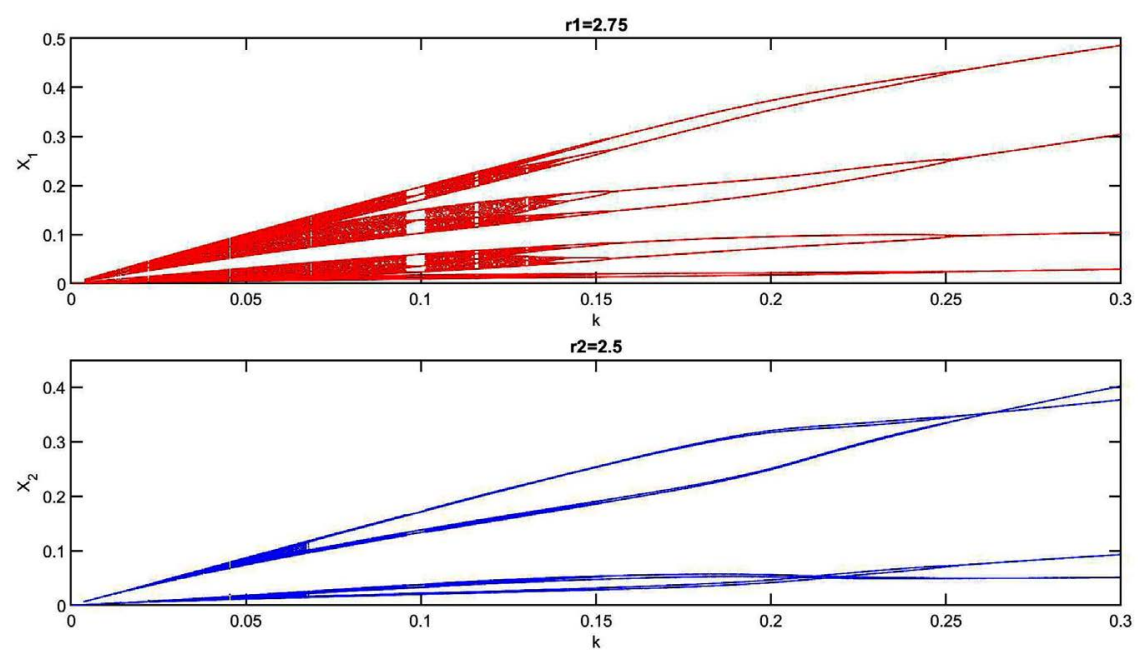

Figure 3. Bifurcation diagram of system (4.1) when $r_{1}=2.75$ and $r_{2}=2.5$. 

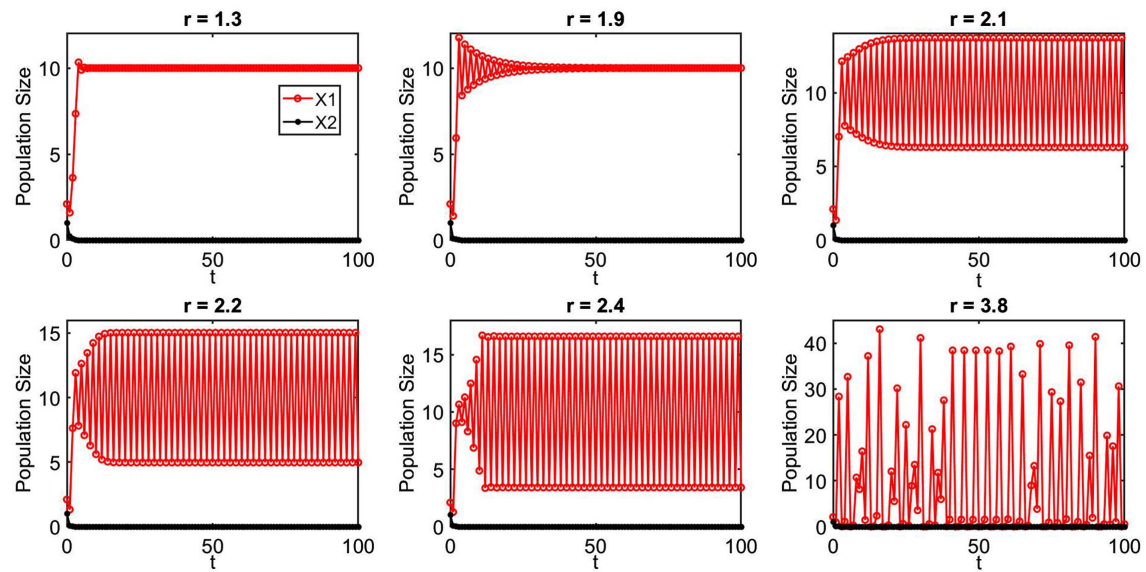

Figure 4. Evolution of host population $X_{1}$ and its coupled $X_{2}$ in time for system (4.1) when $k=10$.

Lemma 4.3 Assume that the conditions of the first part of the proposition (4.2) are satisfied. The fixed point $Z^{*}=\left(X_{1}^{*}, X_{2}^{*}\right)$ of map $F$ is called snap-back repeller if there exists a point $Z_{0}=\left(X_{1}, X_{2}\right)$ in the neighborhood of $Z^{*}$ such that $Z_{0} \neq Z^{*}, F\left(Z_{0}\right)=Z^{*},\left|\operatorname{det}\left(\left.J\right|_{\left(X_{1}, X_{2}\right)}\right)\right| \neq 0$, that is to say, at first, the following system of equations has a unique solution

$$
\left\{\begin{array}{l}
X_{1}^{*}=X_{1} \exp \left(r_{1}\left(1-\frac{X_{1}}{k}-X_{2}\right)\right) \\
X_{2}^{*}=X_{2} \exp \left(r_{2}\left(1-\frac{X_{2}}{k}-X_{1}\right)\right)
\end{array}\right.
$$

and

$$
k^{2}-k\left(r_{2} X_{2}+r_{1} X_{1}+r_{1} r_{2} X_{1} X_{2}\right)+r_{1} r_{2} X_{1} X_{2} \neq 0
$$

Then $Z^{*}$ for some parameter values $\left(r_{1}, r_{2}\right)$ and $k$, is a snap-back repeller for map (5).

Proof. From $\left|\operatorname{det}\left(\left.J\right|_{\left(X_{1}, X_{2}\right)}\right)\right| \neq 0$, we have:

$$
\frac{\partial g_{1}}{\partial X_{1}} \frac{\partial g_{2}}{\partial X_{2}}-\frac{\partial g_{1}}{\partial X_{2}} \frac{\partial g_{2}}{\partial X_{1}} \neq 0
$$

which

$$
\left(\left(1-\frac{r_{1} X_{1}}{k}\right)\left(1-\frac{r_{2} X_{2}}{k}\right)-r_{1} r_{2} X_{1} X_{2}\right) \mathrm{e}^{\left(r_{1}\left(1-\frac{X_{1}}{k}-X_{2}\right)+r_{2}\left(1-\frac{X_{2}}{k}-X_{1}\right)\right)} \neq 0
$$

and it gives us

$$
k^{2}-k\left(r_{2} X_{2}+r_{1} X_{1}+r_{1} r_{2} X_{1} X_{2}\right)+r_{1} r_{2} X_{1} X_{2} \neq 0
$$

Therefore, any solution $Z^{*}=\left(X_{1}^{*}, X_{2}^{*}\right) \neq\left(X_{1}, X_{2}\right)=Z_{0}$ of system (20) which satisfies the first part of the proposition (4.2) and (21), is snap-back repeller for system (5). 
Theorem 4.4 Under the assumptions of the first part of proposition (4.2) and lemma (4.3), the map (4.1) is chaotic in the sense of Li-York, which means that. There exist 1) a positive integer $N$, such that map (4.1) has a point of period $p$, for each integer $p \geq N, 2)$ a scrambled set of $F$, i.e., an uncountable set $S$ containing no periodic points of $F$, such that

a) $F(S) \subset S$,

b) $\limsup _{n \rightarrow \infty}\left\|F^{n}(x)-F^{n}(y)\right\|>0$, for all $x, y \in S$, with $x \neq y$,

c) $\limsup _{n \rightarrow \infty}\left\|F^{n}(x)-F^{n}(y)\right\|>0$, for all $x \in S$ and periodic point $y$ of $f$,

3) an uncountable subset $S_{0}$ of $S$, such that $\liminf _{n \rightarrow \infty}\left\|F^{n}(x)-F^{n}(y)\right\|=0$, for every $x, y \in S_{0}$.

Proof. By theorem (1.3).

\section{Conclusion}

Studying the evolution of population models and complex dynamics of competitive models has attracted many researchers during several past decades. In this paper, we studied the complex dynamics of a two-species Ricker model which consists of four different biological parameters. We explored the stability of the origin and two other boundary fixed points using local stability theorem. Also, we provided the condition under which the solutions are bounded. We have seen that this model undergoes period doubling bifurcation but it does not show Neimark-Sacker bifurcation. We used the persistence theory to reveal the global behavior of system and we discovered the persistence of the system for two boundary fixed points. Afterward, we changed the model to a specific case with only three biological parameters and we discussed about the local stability of extinction and boundary fixed points of the system. Moreover, we discovered the chaotic dynamics of the new model using Marotto theorem. As we discussed, Marotto theorem is a rigorous theorem to study chaotic dynamics for systems with higher dimensions and can be used to study the chaotic dynamics of competitive models. We presented the conditions under which the new system undergoes snap-back repeller and as a result, it is chaotic in the sense of Li-York. Finally, we used bifurcation diagram to demonstrate the interesting dynamics of new system and the role of biological parameters $r$ and $k$ in appearance of different types of complicated dynamics. The new system has the same number of fixed points as the first system and the bifurcation analysis displayed the same qualitative dynamics for both species as we expected.

\section{Conflicts of Interest}

The authors declare no conflicts of interest regarding the publication of this paper.

\section{References}

[1] Liu, H., Zhang, K., Ye, Y., Wei, Y.M. and Ma, M. (2019) Dynamic Complexity and Bifurcation Analysis of a Host-Parasitoid Model with Allee Effect and Holling Type III Functional Response. Advances in Difference Equations, 2019, Article No.: 507. 
https://doi.org/10.1186/s13662-019-2430-8

[2] Smith, H.L. (1998) Planar Competitive and Cooperative Difference Equations. Journal of Difference Equations and Applications, 3, 335-357. https://doi.org/10.1080/10236199708808108

[3] Lus, R., Elaydi, S. and Oliveira, H. (2011) Stability of a Ricker-Type Competitive Model and the Competitive Exclusion Principle. Journal of Difference Equations and Applications, 5, 636-660. https://doi.org/10.1080/17513758.2011.581764

[4] Gyllenberg, M., Jiang, J. and Niu, L. (2019) A Note on Global Stability of ThreeDimensional Ricker Models. Journal of Difference Equations and Applications, 25, 142-150. https://doi.org/10.1080/10236198.2019.1566459

[5] Wu, D.Y. and Zhang, H. (2014) Bifurcation Analysis of a Two-Species Competitive Discrete Model of Plankton Allelopathy. Advances in Difference Equations, 2014, Article No.: 70. https://doi.org/10.1186/1687-1847-2014-70

[6] Azizi, T. and Kerr, G. (2020) Chaos Synchronization in Discrete-Time Dynamical Systems with Application in Population Dynamics. Journal of Applied Mathematics and Physics, 8, 406-423. https://doi.org/10.4236/jamp.2020.83031

[7] Azizi, T. and Kerr, G. (2020) Synchronized Cycles of Generalized Nicholson-Bailey Model. American Journal of Computational Mathematics, 10, 147-166.

https://doi.org/10.4236/ajcm.2020.101009

[8] Li, T.-Y. and Yorke, J.A. (1975) Period Three Implies Chaos. The American Mathematical Monthly, 82, 985-992. https://doi.org/10.1080/00029890.1975.11994008

[9] Marotto, F.R. (1978) Snap-Back Repellers Imply Chaos in Rn. Journal of Mathematical Analysis and Applications, 63, 199-223. https://doi.org/10.1016/0022-247X(78)90115-4

[10] Marotto, F.R. (1979) Chaotic Behavior in the Hénon Mapping. Journal of Mathematical Analysis and Applications, 68, 187-194. https://doi.org/10.1007/BF01418128

[11] Marotto, F.R. (2005) On Redefining a Snap-Back Repeller. Chaos, Solitons \& Fractals, 25, 25-28. https://doi.org/10.1016/j.chaos.2004.10.003

[12] Lin, W., Ruan, J. and Zhao, W.R. (2002) On the Mathematical Clarification of the Snap-Back-Repeller in High-Dimensional Systems and Chaos in a Discrete Neural Network Model. International Journal of Bifurcation and Chaos, 12, 1129-1139. https://doi.org/10.1142/S0218127402004966

[13] Li, C.P. and Chen, G.R. (2003) An Improved Version of the Marotto Theorem. Chaos, Solitons \& Fractals, 18, 69-77. https://doi.org/10.1016/S0960-0779(02)00605-7

[14] Gardini, L. (1994) Homoclinic Bifurcations in $n$-Dimensional Endomorphisms, Due to Expanding Periodic Points. Nonlinear Analysis. Theory, Methods \& Applications, 23, 1039-1089. https://doi.org/10.1016/0362-546X(94)90198-8

[15] Gardini, L., Sushko, I., Avrutin, V. and Schanz, M. (2011) Critical Homoclinic Orbits Lead to Snap-Back Repellers. Chaos, Solitons \& Fractals, 44, 433-449. https://doi.org/10.1016/j.chaos.2011.03.004

[16] Wiggins, S. (2003) Introduction to Applied Nonlinear Dynamical Systems and Chaos. Princeton University Press, Springer Science \& Business Media, Princeton, 2.

[17] Balibrea, F. and Cascales, A. (2012) Li-Yorke Chaos in Perturbed Rational Difference Equations. International Conference on Difference Equations and Applications, 44, 49-61. https://doi.org/10.1007/978-3-662-52927-0_4

[18] Panchuk, A. (2016) Some Aspects on Global Analysis of Discrete Time Dynamical 
Systems. In: Qualitative Theory of Dynamical Systems, Tools and Applications for Economic Modelling, Springer, Cham, 161-186. https://doi.org/10.1007/978-3-319-33276-5_2

[19] Smale, S. (2000) Diffeomorphisms with Many Periodic Points. The Collected Papers of Stephen Smale: Volume 2. World Scientific, 44, 636-653.

[20] Moser, J. (2001) Stable and Random Motions in Dynamical Systems: With Special Emphasis on Celestial Mechanics. Princeton University Press, Princeton, 1. https://doi.org/10.1515/9781400882694

[21] Robinson, C. (1998) Dynamical Systems: Stability, Symbolic Dynamics, and Chaos. CRC Press, Boca Raton.

[22] Guckenheimer, J. and Holmes, P. (1984) Nonlinear Oscillations, Dynamical Systems and Bifurcations of Vector Fields. Journal of Applied Mechanics, 51, 947. https://doi.org/10.1115/1.3167759

[23] Shilnikov, L.P. (1970) A Contribution to the Problem of the Structure of an Extended Neighborhood of a Rough Equilibrium State of Saddle-Focus Type. Mathematics of the USSR-Sbornik, 10, 91. https://doi.org/10.1070/SM1970v010n01ABEH001588

[24] Shilnikov, L.P., Shilnikov, A.L., Turaev, D.V. and Chua, L.O. (2001) Methods Of Qualitative Theory In Nonlinear Dynamics (Part II). World Scientific, Singapore, New Jersey, London, Hong Kong. https://doi.org/10.1142/4221

[25] Mira, C. (1987) Chaotic Dynamics: From the One-Dimensional Endomorphism to the Two-Dimensional Diffeomorphism. World Scientific, Singapore. https://doi.org/10.1142/0413

[26] Ricker, W.E. (1954) Stock and Recruitment. Journal of the Fisheries Board of Canada, 11, 559-623. https://doi.org/10.1139/f54-039

[27] Azizi, T., et al. (2015) Dynamics of a Discrete-Time Plant-Herbivore Model. Caspian Journal of Mathematical Sciences (CJMS), 4, 241-256.

[28] Smith, H.L. and Thieme, H.R. (2011) Dynamical Systems and Population Persistence. American Mathematical Society, Providence, 118.

[29] Kong, X.Z., Chen, L.P. and Yang, W.S. (2010) Note on the Persistent Property of a Discrete Lotka-Volterra Competitive System with Delays and Feedback Controls. Advances in Difference Equations, 2010, Article No.: 249364. https://doi.org/10.1155/2010/249364

[30] Thieme, H.R. (1993) Persistence under Relaxed Point-Dissipativity (with Application to an Endemic Model). SIAM Journal on Mathematical Analysis, 24, 407-435. https://doi.org/10.1137/0524026

[31] Hirsch, M.W., Smith, H.L. and Zhao, X.Q. (2001) Chain Transitivity, Attractivity, and Strong Repellors for Semidynamical Systems. Journal of Dynamics and Differential Equations, 13, 107-131. https://doi.org/10.1023/A:1009044515567

[32] Kulenovic, M.R.S. and Ladas, G. (2001) Dynamics of Second Order Rational Difference Equations: With Open Problems and Conjectures. Chapman and Hall/CRC, New York. https://doi.org/10.1201/9781420035384 